\title{
Exomic and Epigenomic Analyses in a Pair of Monozygotic Twins Discordant for Cryptorchidism
}

\author{
Peng Lu, ${ }^{1}, *$ Pengyun Wang, ${ }^{2, *}$ Lianbing Li, ${ }^{3}$ Chengqi Xu, ${ }^{4}$ Jing Crystal Liu, ${ }^{5}$ Xiangqian Guo, ${ }^{6}$ Dawei He, ${ }^{1}$ \\ Huizhe Huang, ${ }^{5}$ and Zhi Cheng ${ }^{5}$ \\ ${ }^{1}$ Department of Urology, Children's Hospital of Chongqing Medical University, Ministry of Education Key Laboratory of \\ Child Development and Disorders, Chongqing, PR China \\ ${ }^{2}$ Department of Clinical Laboratory, Liyuan Hospital, Huazhong University of Science and Technology, Wuhan, PR China \\ ${ }^{3}$ Key Laboratory of Birth Defects and Reproductive Health of the National Health and Family Planning Commission \\ (Chongqing Population and Family Planning Science and Technology Research Institute), Chongqing, PR China \\ ${ }^{4}$ College of Life Science and Technology and Center for Human Genome Research, Key Laboratory of Molecular \\ Biophysics of the Ministry of Education, Huazhong University of Science and Technology, Wuhan, PR China \\ ${ }^{5}$ School of Basic Medical Sciences, Chongqing Medical University, Chongqing, PR China \\ ${ }^{6}$ Department of Preventive Medicine, Medical School, Henan University, Kaifeng, PR China
}

\begin{abstract}
Cryptorchidism represents one of the most common human congenital anomalies. In most cases, its etiology remains unclear and seems to be multifactorial. In the present study, a pair of monozygotic twins discordant for cryptorchidism was identified. Twin zygosity was confirmed by microsatellite genotyping. Whole exome sequencing and methylated DNA immunoprecipitation sequencing (MeDIP-Seq) of DNA extract from leucocytes were performed to, respectively, evaluate their exomes and epigenomes. No differences in exome sequencing data were found between the twins after validation. MeDIP-Seq analysis detected 5,410 differentially hypermethylated genes and 2,383 differentially hypomethylated genes. Bioinformatic analysis showed that these genes belonged to several biological processes and signaling pathways, including regulation of actin cytoskeleton, which has been previously implicated in the etiology of cryptorchidism. The findings of the present study suggest that non-genetic factors might contribute to the pathogenesis of cryptorchidism.
\end{abstract}

Keywords: cryptorchidism, twins, exome sequencing, methylated DNA immunoprecipitation sequencing

Cryptorchidism, defined as the failure of one or both testes to descend into the scrotum, represents one of the most common human congenital anomalies. While it can be part of hundreds of congenital malformation syndromes, it mainly appears as an isolated condition (Barthold et al., 2016). The major clinical consequences of this abnormality include reduced fertility in adulthood and an increased risk for testicular cancer (Hutson et al., 2010).

The etiology of non-syndromic cryptorchidism remains unknown in most cases and seems to be multifactorial. Familial clustering and increased prevalence in first-degree relatives suggests that genetics may play a role in the development of this disease (Elert et al., 2003; Schnack et al., 2008). Mutations in the gene encoding insulin-like factor 3 , which regulates the transabdominal phase of testicular descent, and in its receptor, RXFP2, have been linked to approximately $5 \%$ of cryptorchidism cases (Foresta et al., 2008). However, analysis of the HOXA10 gene failed to show consistent results (Cheng et al., 2017; Kolon et al.,
1999). Similarly, candidate gene studies showed conflicting associations of the androgen receptor $(A R)$ and estrogen receptor 1 (ESR1) genes with cryptorchidism (Barthold et al., 2016). Recent genome-wide association studies (GWASs) have identified several loci in suggestive association with cryptorchidism, although none of these have shown genome-wide significance (Barthold et al., 2015). The results of the previous studies are thus suggestive of the complex genetic architecture that is associated with cryptorchidism.

RECeIVed 16 January 2017; ACCePted 2 May 2017. First published online 13 June 2017.

ADDRESS FOR CORRESPONDENCE: Zhi Cheng, School of Basic Medical Sciences, Chongqing Medical University, NO.1 Yixueyuan Road, Yuzhong District, Chongqing 400016, PR China. E-mail: zcheng@cqmu.edu.cn

* These authors contributed equally to the work. 
Maternal and environmental factors are also believed to contribute to the etiology of cryptorchidism. Maternal smoking and gestational diabetes increase the risk for this disease (Zhang et al., 2015). Furthermore, pieces of evidence from animal models and human epidemiological studies suggest that exposure to endocrine-disrupting chemicals (EDCs), such as isphenol A, phthalates, and pesticides, may be causally associated with cryptorchidism (Virtanen \& Adamsson, 2012). EDCs act as antiandrogens or estrogens that disrupt normal hormonal stimulation during testicular descent. Because these exogenous factors have the potential to elicit additional epigenetic effects, it is highly likely that epigenetic alterations are also involved in the etiology of cryptorchidism (Guerrero-Bosagna \& Skinner, 2014).

Monozygotic (MZ) twins offer the ideal model to dissect the relative contributions of genetic and environmental factors in the pathogenesis of multifactorial diseases (Baranzini et al., 2010; Jin et al., 2014). It is generally considered that MZ twins are identical in genotype, and phenotypic discordance is ascribed to environmental factors. Previous studies have detected genetic and epigenetic differences between MZ twins (Bruder et al., 2008; Yuan et al., 2014). Genetic differences may result from very early posttwinning mutational events, and epigenetic changes are driven by environmental factors (Heijmans et al., 2008; Kondo et al., 2002). In the present study, genomic and epigenomic differences were analyzed in a pair of $\mathrm{MZ}$ twins discordant for cryptorchidism for the first time, aiming to identify the specific alterations responsible for cryptorchidism.

\section{Materials and Methods}

\section{Case Report}

The patient was an 11-month-old boy who had undergone orchidopexy at the Children's Hospital of Chongqing Medical University because of bilateral undescended testes, which were localized within the inguinal canal. He had a healthy MZ twin brother who showed normal testicular descent. The twins were delivered by the Cesarean section after 39 weeks of gestation to healthy, non-consanguineous parents. The patient had no family history and no other clinical symptoms except for cryptorchidism. A written informed consent was obtained from the parents of the twins. The study protocol was in accordance with the tenets of the Declaration of Helsinki and approved by the institutional ethnics committee of the Children's Hospital of Chongqing Medical University.

\section{Genomic DNA Extraction}

Approximately, $5 \mathrm{~mL}$ of peripheral blood was obtained from both twins using a heparin anticoagulation tube. Genomic DNA was extracted from leukocytes using a DNA isolation kit (Tiangen, Beijing, China) according to the protocol provided by the manufacturer. DNA samples were quantified using a NanoDrop 2000 spectrophotometer (Thermo Fisher Scientific Inc., Waltham, MA). Purified genomic DNA was stored at $-80^{\circ} \mathrm{C}$ until use.

\section{Zygosity Analysis}

To confirm that the twins were MZ, amelogenin and 21 autosomal short tandem repeat (STR) profiles were obtained using AGCU EX22 STR Kit (AGCU ScienTech Inc., Wuxi, China) according to the manufacturer's protocols. All of the amplification reactions were performed in a GeneAmp PCR system 9700 (Perkin Elmer, Norwalk, CT). The amplified products were run on an ABI 3130 Genetic Analyzer (Applied Biosystems, Foster City, CA), and the data were analyzed using GeneMapper ID v3.2 software (ABI).

\section{Whole Exome Sequencing (WES) and Data Analysis}

Genomic DNA was randomly broken into manageable fragments and subjected to library preparation. Paired-end libraries with a span size of $\sim 300$ bp were adopted for exome sequencing. Exome enrichment was performed using the SureSelect Human All Exon $50 \mathrm{Mb}$ kit (Agilent Technologies, Santa Clara, CA). The enriched libraries were sequenced using the Illumina HiSeq Xten sequencing system (Illumina, San Diego, CA), and 150-bp paired-end reads were generated. The sequencing data were mapped to the hg19 human reference sequence using the BurrowsWheeler Aligner (BWA) program (Li \& Durbin, 2010). PCR duplicates were marked using the Picard program. Genome Analysis Toolkit (GATK) was then used for indel realignment and base quality recalibration (McKenna et al., 2010). Single nucleotide variants (SNVs) and small insertions and deletions (InDels) were called and recalibrated using GATK. Variants were annotated using the ANNOVAR (Wang et al., 2010).

To help identify candidate mutations, the following variants were filtered out: (1) synonymous substitutions, intergenic and intronic variants, and variants located within untranslated regions; (2) variants present in dbSNP142 and 1,000 Genomes databases; (3) variants with a minor allele frequency of $>1 \%$; and (4) variants with the phred-scaled quality score of $<30$ or a read depth of $<4$. In addition, only variants specific to the affected twin were further analyzed.

\section{Validation of Discordant Variation}

A total of 37 discordant variations identified by Whole Exome Sequencing (WES) were selected for amplification from genomic DNA by polymerase chain reaction (PCR) using specific primer pairs (Table S1). The resulting PCR products were purified and sequenced on an ABI 3730XL sequencer $(\mathrm{ABI})$ using a BigDye Terminator Cycle Sequencing Kit (ABI). 


\section{Methylated DNA Immunoprecipitation Sequencing} (MeDIP-Seq) and Bioinformatic Analyses

Genomic DNA samples were fragmented at sizes ranging from 200 bp to $900 \mathrm{bp}$ with a Bioruptor sonicator (Diagenode, Liège, Belgium). Approximately, 800 ng of sonicated DNA was end-repaired, attached with polyA tails, and ligated to single-end adapters following the standard Illumina genomic DNA protocol. After agarose size-selection to remove unligated adapters, the adaptor-ligated DNA was used for immunoprecipitation using a monoclonal antibody against 5-methylcytidine (Diagenode). MeDIP enrichment was performed according to the previously described protocol (Tao et al., 2015). About $150 \mathrm{ng}$ of the affected twin's sonicated and adaptor-ligated DNA that did not undergo immunoprecipitation was used as input. The input and antibody-enriched DNA was amplified by PCR using the single-end Illumina PCR primers and then purified by agarose gel electrophoresis. The products were then quantified on an Agilent 2100 Analyzer (Agilent) and subjected to qPCR qualification. Sequencing was performed on Illumina HiSeq 2000 using the TruSeq Rapid SBS Kit (Cat. \# FC-402-4001, Illumina).

High-quality clean reads were aligned to the human reference genome (hg19) using SOAP2 (Version 2.21) with no more than 2 bp mismatches (Li et al., 2009). Only uniquely mapped reads were further analyzed. The distribution of reads in chromosomes and in the different genome components was analyzed. Genome-wide methylation peak scanning was performed using MACS V1.4.2 (Zhang et al., 2008). The region with a $p$ value of $<10^{-5}$ was defined as a peak. The distribution of peaks in different genome components was also analyzed. The genomic differentially methylated regions (DMRs) between the twins were detected using a previously described method (Kim et al., 2016), except that the significance cutoff of $p$ value for the chi-square statistics and false discovery rate (FDR) was 0.01 . The genes with at least one region overlapping with DMRs were considered differentially methylated genes. All differentially methylated genes were then subjected to Gene Ontology (GO) and the Kyoto Encyclopedia of Genes and Genomes (KEGG) pathway analysis using the DAVID bioinformatics resources (Huang et al., 2009).

\section{Results}

\section{Zygosity Analysis}

STR analysis revealed that the twins had the identical alleles for all 21 microsatellite markers (Table 1), thereby confirming that they were indeed MZ.

\section{WES Analysis and Variant Validation}

WES was performed on genomic DNA of the twins to identify the underlying genetic mutations. An average of 12.44 $\mathrm{Gb}$ of effective sequence data was obtained per individual. The mean sequencing depth of the target region of each

\section{TABLE 1}

Short Tandem Repeat Typing Results of the Patient and Twin Brother

\begin{tabular}{|c|c|c|c|c|}
\hline \multirow[b]{2}{*}{ Marker } & \multicolumn{2}{|c|}{ Affected twin } & \multicolumn{2}{|c|}{ Healthy twin } \\
\hline & Allele 1 & Allele 2 & Allele 1 & Allele 2 \\
\hline AMEL & $x$ & $Y$ & $x$ & $Y$ \\
\hline D3S1358 & 17 & 17 & 17 & 17 \\
\hline D13S317 & 8 & 8 & 8 & 8 \\
\hline D7S820 & 8 & 12 & 8 & 12 \\
\hline D16\$539 & 9 & 11 & 9 & 11 \\
\hline Penta E & 5 & 11 & 5 & 11 \\
\hline D2S441 & 11.3 & 14 & 11.3 & 14 \\
\hline TPOX & 9 & 12 & 9 & 12 \\
\hline TH01 & 7 & 9 & 7 & 9 \\
\hline D2S1338 & 19 & 23 & 19 & 23 \\
\hline CSF1PO & 12 & 14 & 12 & 14 \\
\hline Penta D & 9 & 13 & 9 & 13 \\
\hline D10S1248 & 15 & 15 & 15 & 15 \\
\hline D19S443 & 13 & 15 & 13 & 15 \\
\hline vWA & 14 & 18 & 14 & 18 \\
\hline D21S11 & 29 & 31.2 & 29 & 31.2 \\
\hline D18S51 & 13 & 16 & 13 & 16 \\
\hline D6S1043 & 10 & 18 & 10 & 18 \\
\hline D8S1179 & 16 & 17 & 16 & 17 \\
\hline D5S818 & 7 & 12 & 7 & 12 \\
\hline D12S391 & 18 & 22 & 18 & 22 \\
\hline FGA & 22 & 24 & 22 & 24 \\
\hline
\end{tabular}

individual was $160 \times$ and $161 \times$, respectively. Furthermore, $>98 \%$ of the target region in both samples showed at least $20 \times$ coverage (Table S2).

Bioinformatics analysis identified a total of 97,692 SNVs and 15,678 InDels in the affected twin, whereas 99,249 SNVs and 15,976 InDels were detected in the healthy twin (Tables S3 and S4). Given the discordant phenotypes of the twins, 1,368 SNVs and 917 InDels specific to the affected twin were identified. Non-synonymous variations, splice site variations, and InDels were selected for further analysis. After applying the stringent filtering criteria, approximately 35 discordant SNVs and 11 InDels were obtained (Figure 1; Table S5).

All 35 discordant SNVs were selected to validate by the Sanger sequencing. However, none of these variations could be confirmed. While 34 of these SNVs were found to be false-positive calls, an SNV was turned out to exist in both twins. Two InDels in genes that were expressed in fetal testis according to the GeneCards database (http://www.genecards.org/) were selected for validation, neither of which was validated. And these InDels were proved to be false-positive calls.

\section{Genome-Wide DNA Methylation Analysis}

MeDIP-Seq was performed to detect epigenomic differences between the twins. A total of 33,033,859 clean reads were generated per sample, $69.9 \%$ of which could be uniquely aligned to the human genome (Table S6). In addition, the reads were detected in most chromosomal regions (Figure S1). The analysis of the read distribution in different genome components revealed that these were mostly located in intronic regions, which contained $39 \%$ of the 
97,692 SNVs and 15,678 InDels

Select variants involved in the affected twin and absent in the other twin

1,368 SNVs and 917 InDels

Remove variants in intergenic, intronic and untranslated regions

120 SNVs and 23 InDels

Remove Synonymous variants

88 SNVs and 23 InDels

Remove variants with phred-scaled quality score $<30$ or reads depth $<4$

62 SNVs and 15 InDels

Remove variants with MAF $>1 \%$ in public

database

35 SNVs and 11 InDels

\section{FIGURE 1}

Illustration of the variant filtering procedure. Note: SNV = single nucleotide variation, InDels = small insertions and deletions; MAF $=$ minor allele frequency.

unique reads (Figure S2A). Within genic regions, the number of reads dramatically decreased within the transcription start sites of both samples, whereas a gradual increase was observed in intragenic regions (Figure S2B).

Whole genome methylated peak scanning revealed approximately 51,647 peaks in the affected twin and 11,154 peaks in the healthy twin, covering $2.14 \%$ and $0.36 \%$ of the genome, respectively (Table S7). The majority of the peaks were present in intronic regions, followed by coding sequences (CDSs; Figure S3). The differentially methylated genes between the twins were then determined. A total of 5,410 genes were differentially hypomethylated, and 2,383 genes were differentially hypermethylated in the affected twin compared to the healthy twin (Supplementary file 2). Most of these genes were differentially methylated in the intronic regions, followed by the CDSs (Figure S4).

GO analysis was performed to determine biologically functional relationships among differentially methylated genes. In addition, GO terms enriched in the biological process were identified. The results showed that eight hypermethylated genes were related to microtubule-based movement (FDR $<0.05$; Supplementary file 3 ). The hypomethylated genes were mainly correlated with 36 categories and 1,056 genes were involved (FDR $<0.05$; Supplementary file 4). The top 15 categories included protein phosphorylation, cell migration, positive regulation of GTPase activity, cell adhesion, and protein autophosphorylation (Figure 2A).

KEGG pathway analysis revealed the significant pathways that were associated with differentially methylated genes. The hypermethylated genes were not observed to tightly associate with any pathways. The hypomethylated genes were significantly enriched in 25 pathways and 408 genes were involved (FDR $<0.05$; Supplementary file 5). The top 15 significant pathways included calcium signaling, Rap1 signaling, axon guidance, cAMP signaling, and glutamatergic synapse (Figure 2B).

\section{Discussion}

In the present study, no genetic differences were detected in MZ twins discordant for cryptorchidism. The results coincided with the findings of earlier similar studies, in which no genetic discordances were found in MZ twins discordant for several disorders, including renal agenesis (Jin et al., 2014), cataract (Wei et al., 2015), VACTERL association (Solomon et al., 2013), hypothyroidism (Magne et al., 2015), heart defects, and diaphragmatic hernia (Zhang et al., 2016). Our findings provide additional evidence that genetic mutations are not a common cause for phenotypic discordance among MZ twins.

MeDIP-seq is a cost-effective method in establishing the global DNA methylation patterns of the human genome. Using this methylome technology, epigenetic changes were detected in the current twin pair, thereby suggesting that the discordant phenotype might have resulted from alterations in DNA methylation patterns. Although MZ twins share a single uterus, they might be exposed to different intrauterine environments such as uneven blood supply and different nutritional conditions (Czyz et al., 2012). The epigenetic changes triggered by non-shared early exposures might thus affect the expression of regulatory genes during embryogenesis, thereby leading to a particular disease phenotype.

The epigenetic alterations underlying the etiology of cryptorchidism are poorly understood to date. Our comparison of DNA methylation profiles between the twins revealed that differentially methylated genes were enriched with several significant biological processes and pathways. Among these, the actin cytoskeleton pathways, which were identified by both GO and KEGG pathway analysis (Figure 2), require further investigation. In previous GWAS on non-syndromic cryptorchidism, pathway analysis of suggestive signals also identified various genetic loci that were related to cytoskeleton-dependent functions (Barthold et al., 2015). Moreover, alterations in the expression of cytoskeleton-related genes were observed in a rat strain with congenital cryptorchidism (Barthold et al., 2008). Therefore, despite the need to confirm our methylation results in additional samples, the present study offers the clues that aberrations in the methylation patterns of cytoskeleton-associated genes may contribute to the pathogenesis of cryptorchidism.

To the best of our knowledge, this is the first genomewide study to investigate the genomic variations and epigenetic differences in discordant $\mathrm{MZ}$ twins with 

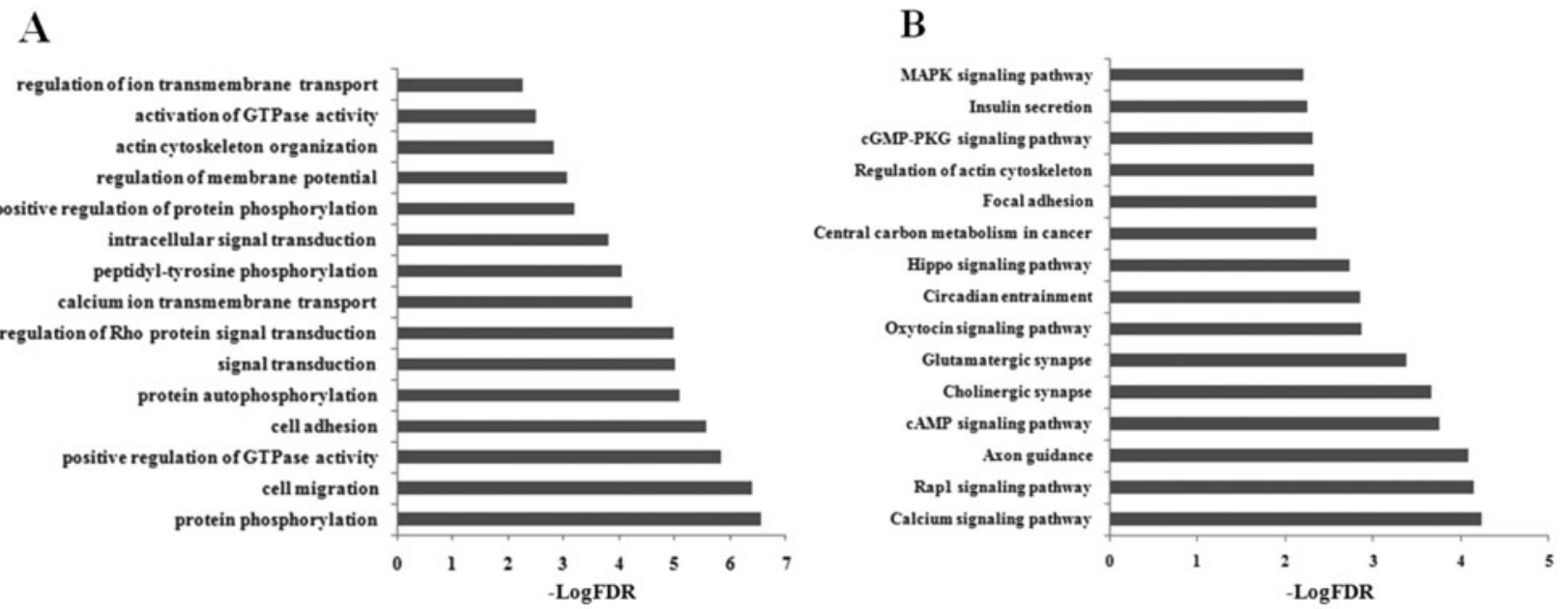

\section{FIGURE 2}

Gene ontology and pathway analysis of hypomethylated genes. A. Top 15 biological processes involving hypomethylated genes. B. Top 15 significant pathways involving hypomethylated genes.

cryptorchidism. Previous studies have detected gene mutations in only a fraction of cryptorchidism cases. It has also been suggested that the pathogenesis of cryptorchidism is multifactorial, which include genetic and environmental factors. In the present study, no genomic mutations but epigenetic differences were detected in discordant MZ twins. Our findings provide evidence for the multifactorial nature of cryptorchidism, particularly non-genetic factors, thereby providing new insights into the pathogenesis of cryptorchidism.

The present study has two limitations. First, only one pair of MZ twins was investigated. More pairs of discordant MZ twins should be recruited in the future to confirm the findings of this study. Second, DNA methylation is tissue specific. The methylation patterns in leukocytes were examined in the present study, whereas cryptorchidism is a developmental defect that involves the gubernaculum and testis, which are generally difficult to obtain from human subjects. As a result, animal models should be used to verify the epigenetic alterations identified in this study.

In summary, we have performed WES and MeDIPSeq analyses in a pair of MZ twins discordant for cryptorchidism. No genomic mutations were detected, whereas epigenetic alterations were observed, thereby suggesting that non-genetic factors may contribute to the pathogenesis of cryptorchidism.

\section{Disclosure of Interest}

None.

\section{Supplementary material}

To view supplementary material for this article, please visit https://doi.org/10.1017/thg.2017.33

\section{Acknowledgments}

This work was supported by the National Natural Science Foundation of China (grant number 81300463) and the Natural Science Foundation Project of Chongqing (grant number cstc2012jjjq10001).

\section{References}

Baranzini, S. E., Mudge, J., van Velkinburgh, J. C., Khankhanian, P., Khrebtukova, I., Miller, N. A., ... Kingsmore, S. F. (2010). Genome, epigenome and RNA sequences of monozygotic twins discordant for multiple sclerosis. Nature, 464, 1351-1356.

Barthold, J. S., McCahan, S. M., Singh, A. V., Knudsen, T. B., Si, X., Campion, L., \& Akins, R. E. (2008). Altered expression of muscle- and cytoskeleton-related genes in a rat strain with inherited cryptorchidism. Journal of Andrology, 29, 352-366.

Barthold, J. S., Reinhardt, S., \& Thorup, J. (2016). Genetic, maternal, and environmental risk factors for cryptorchidism: An update. European Journal of Pediatric Surgery, 26, 399408.

Barthold, J. S., Wang, Y., Kolon, T. F., Kollin, C., Nordenskjöld, A., Olivant Fisher, A., ... Devoto, M. (2015). Pathway analysis supports association of nonsyndromic cryptorchidism with genetic loci linked to cytoskeleton-dependent functions. Human Reproduction, 30, 2439-2451.

Bruder, C. E., Piotrowski, A., Gijsbers, A. A., Andersson, R., Erickson, S., Diaz de Ståhl, T., ... Dumanski, J. P. (2008). Phenotypically concordant and discordant monozygotic twins display different DNA copy-number-variation profiles. American Journal of Human Genetics, 82, 763-771.

Cheng, Z., Wang, M., Xu, C., Pei, Y., Liu, J. C., Huang, H., ... Lu, P. (2017). Mutational analysis of HOXA10 gene in Chinese patients with cryptorchidism. Andrologia, 49(1). doi:10.1111/and.12592. 
Czyz, W., Morahan, J. M., Ebers, G. C., \& Ramagopalan, S. V. (2012). Genetic, environmental and stochastic factors in monozygotic twin discordance with a focus on epigenetic differences. BMC Medicine, 10, 93.

Elert, A., Jahn, K., Heidenreich, A., \& Hofmann, R. (2003). The familial undescended testis. Klinische Padiatrie, 215, 40-45.

Foresta, C., Zuccarello, D., Garolla, A., \& Ferlin, A. (2008). Role of hormones, genes, and environment in human cryptorchidism. Endocrine Reviews, 29, 560-580.

Guerrero-Bosagna, C., \& Skinner, M. K. (2014). Environmentally induced epigenetic transgenerational inheritance of male infertility. Current Opinion in Genetics \& Development, 26, 79-88.

Heijmans, B. T., Tobi, E. W., Stein, A. D., Putter, H., Blauw, G. J., Susser, E. S., ... Lumey, L. H. (2008). Persistent epigenetic differences associated with prenatal exposure to famine in humans. Proceedings of the National Academy of Sciences of the United States of America, 105, 17046-17049.

Huang, da W., Sherman, B. T., \& Lempicki, R. A. (2009). Systematic and integrative analysis of large gene lists using DAVID bioinformatics resources. Nature Protocols, 4, 4457.

Hutson, J. M., Balic, A., Nation, T., \& Southwell, B. (2010). Cryptorchidism. Seminars in Pediatric Surgery, 19, 215224.

Jin, M., Zhu, S., Hu, P., Liu, D., Li, Q., Li, Z., ... Chen, X. (2014). Genomic and epigenomic analyses of monozygotic twins discordant for congenital renal agenesis. American Journal of Kidney Diseases, 64, 119-122.

Kim, M. C., Kim, N. Y., Seo, Y. R., \& Kim, Y. (2016). An integrated analysis of the genome-wide profiles of DNA methylation and MRNA expression defining the side population of a human malignant mesothelioma cell line. Journal of Cancer, 7, 1668-1679.

Kolon, T. F., Wiener, J. S., Lewitton, M., Roth, D. R., Gonzales, E. T., \& Lamb, D. J. (1999). Analysis of homeobox gene HOXA10 mutations in cryptorchidism. Journal of Urology, $161,275-280$.

Kondo, S., Schutte, B. C., Richardson, R. J., Bjork, B. C., Knight, A. S., Watanabe, Y., ... Murray, J. C. (2002). Mutations in IRF6 cause Van der Woude and popliteal pterygium syndromes. Nature Genetics, 32, 285-289.

Li, H., \& Durbin, R. (2010). Fast and accurate long-read alignment with Burrows-Wheeler transform. Bioinformatics, 26, 589-595.

Li, R., Yu, C., Li, Y., Lam, T. W., Yiu, S. M., Kristiansen, K., \& Wang, J. (2009). SOAP2: an improved ultrafast tool for short read alignment. Bioinformatics, 25, 1966-1967.

Magne, F., Serpa, R., Van Vliet, G., Samuels, M. E., \& Deladoëy, J. (2015). Somatic mutations are not observed by ex- ome sequencing of lymphocyte DNA from monozygotic twins discordant for congenital hypothyroidism due to thyroid dysgenesis. Hormone Research in Paediatrics, 83, 7985.

McKenna, A., Hanna, M., Banks, E., Sivachenko, A., Cibulskis, K., Kernytsky, A., ... DePristo, M. A. (2010). The genome analysis toolkit: A MapReduce framework for analyzing next-generation DNA sequencing data. Genome Research, 20, 1297-1303.

Schnack, T. H., Zdravkovic, S., Myrup, C., Westergaard, T., Wohlfahrt, J., \& Melbye, M. (2008). Familial aggregation of cryptorchidism-A nationwide cohort study. American Journal of Epidemiology, 167, 1453-1457.

Solomon, B. D., Pineda-Alvarez, D. E., Hadley, D. W., Hansen, N. F., Kamat, A., Donovan, F. X., ... NISC Comparative Sequencing Program. (2013). Exome sequencing and highdensity microarray testing in monozygotic twin pairs discordant for features of VACTERL association. Molecular Syndromology, 4, 27-31.

Tao, Y. F., Fang, F., Hu, S. Y., Lu, J., Cao, L., Zhao, W. L., ... Pan, J. (2015). Hypermethylation of the GATA binding protein 4 (GATA4) promoter in Chinese pediatric acute myeloid leukemia. BMC Cancer, 15, 756.

Virtanen, H. E., \& Adamsson, A. (2012). Cryptorchidism and endocrine disrupting chemicals. Molecular and Cellular Endocrinology, 355, 208-220.

Wang, K., Li, M., \& Hakonarson, H. (2010). ANNOVAR: Functional annotation of genetic variants from high-throughput sequencing data. Nucleic Acids Research, 38, e164.

Wei, T., Sun, H., Hu, B., Yang, J., Qiao, C., \& Yan, M. (2015). Exome sequencing and epigenetic analysis of twins who are discordant for congenital cataract. Twin Research and $\mathrm{Hu}$ man Genetics, 18, 393-398.

Yuan, W., Xia, Y., Bell, C. G., Yet, I., Ferreira, T., Ward, K. J., ... Spector, T. D. (2014). An integrated epigenomic analysis for type 2 diabetes susceptibility loci in monozygotic twins. Nature Communications, 5, 5719.

Zhang, L., Wang, X. H., Zheng, X. M., Liu, T. Z., Zhang, W. B., Zheng, H., \& Chen, M. F. (2015). Maternal gestational smoking, diabetes, alcohol drinking, pre-pregnancy obesity and the risk of cryptorchidism: A systematic review and meta-analysis of observational studies. PLoS One, 10, e0119006.

Zhang, R., Thiele, H., Bartmann, P., Hilger, A. C., Berg, C., Herberg, U., ... Reutter, H. (2016). Whole-exome sequencing in nine monozygotic discordant twins. Twin Research and Human Genetics, 19, 60-65.

Zhang, Y., Liu, T., Meyer, C. A., Eeckhoute, J., Johnson, D. S., Bernstein, B. E., ... Liu, X. S. (2008). Model-based analysis of ChIP-Seq (MACS). Genome Biology, 9, R137. 